\title{
Potential application of a handheld confocal endomicroscope imaging system using a variety of fluorophores in experimental gliomas and normal brain
}

\author{
Nikolay L. Martirosyan, M.D., ${ }^{1}$ Joseph Georges, B.S., ${ }^{4}$ Jennifer M. Eschbacher, M.D., 2 \\ Daniel D. Cavalcanti, M.D. ${ }^{1}$ Ali M. Elhadi, M.D., ${ }^{1}$ Mohammed G. Abdelwahab, B.S., ${ }^{3}$ \\ Adrienne C. Scheck, Ph.D., ${ }^{3}$ Peter Nakaji, M.D., ${ }^{1}$ Robert F. Spetzler, M.D., ${ }^{1}$ \\ and Mark C. Preul, M.D. ${ }^{1}$
}

Divisions of ${ }^{1}$ Neurological Surgery and ${ }^{2}$ Neuropathology, and ${ }^{3}$ Neuro-Oncology Research, Barrow Neurological Institute, St. Joseph's Hospital and Medical Center, Phoenix; and ${ }^{4}$ School of Life Sciences, Arizona State University, Tempe, Arizona

\begin{abstract}
Object. The authors sought to assess the feasibility of a handheld visible-wavelength confocal endomicroscope imaging system (Optiscan 5.1, Optiscan Pty., Ltd.) using a variety of rapid-acting fluorophores to provide histological information on gliomas, tumor margins, and normal brain in animal models.

Methods. Mice $(\mathrm{n}=25)$ implanted with GL261 cells were used to image fluorescein sodium (FNa), 5-aminolevulinic acid (5-ALA), acridine orange (AO), acriflavine (AF), and cresyl violet (CV). A U251 glioma xenograft model in rats $(\mathrm{n}=5)$ was used to image sulforhodamine 101 (SR101). A swine $(\mathrm{n}=3)$ model with AO was used to identify confocal features of normal brain. Images of normal brain, obvious tumor, and peritumoral zones were collected using the handheld confocal endomicroscope. Histological samples were acquired through biopsies from matched imaging areas. Samples were visualized with a benchtop confocal microscope. Histopathological features in corresponding confocal images and photomicrographs of $\mathrm{H} \& \mathrm{E}-$-stained tissues were reviewed.

Results. Fluorescence induced by FNa, 5-ALA, AO, AF, CV, and SR101 and detected with the confocal endomicroscope allowed interpretation of histological features. Confocal endomicroscopy revealed satellite tumor cells within peritumoral tissue, a definitive tumor border, and striking fluorescent cellular and subcellular structures. Fluorescence in various tumor regions correlated with standard histology and known tissue architecture. Characteristic features of different areas of normal brain were identified as well.

Conclusions. Confocal endomicroscopy provided rapid histological information precisely related to the site of microscopic imaging with imaging characteristics of cells related to the unique labeling features of the fluorophores. Although experimental with further clinical trial validation required, these data suggest that intraoperative confocal imaging can help to distinguish normal brain from tumor and tumor margin and may have application in improving intraoperative decisions during resection of brain tumors.

(http://thejns.org/doi/abs/10.3171/2013.11.FOCUS13486)
\end{abstract}

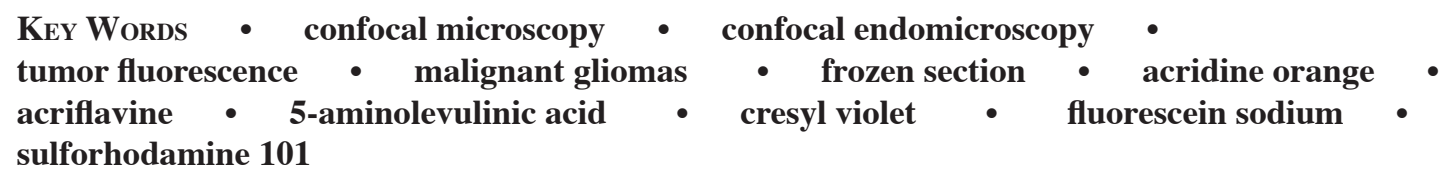

$\mathrm{S}$ URVIVAL of patients with glioma positively correlates with extent of tumor resection..$^{2,9,23,29,33,48}$ Therefore, it is important to distinguish between normal brain and neoplastic tissue intraoperatively to maximize the extent of glioma resection. Routinely used methods of in-

\footnotetext{
Abbreviations used in this paper: $\mathrm{AF}=$ acriflavine hydrochloride; $\mathrm{AO}=$ acridine orange; $\mathrm{BBB}=$ blood-brain barrier; $\mathrm{CV}=$ cresyl violet; $\mathrm{FNa}=$ fluorescein sodium; GFAP = glial fibrillary acidic protein; ICG = indocyanine green; LSM = laser scanning microscope; NIRCE = near-infrared confocal endomicroscope; PpIX = protoporphyrin IX; ROI = region of interest; SR101 = sulforhodamine 101; VWCE = visible-wavelength confocal endomicroscope; 5-ALA = 5-aminolevulinic acid.
}

traoperative diagnosis, such as MRI and ultrasonography, cannot provide spatial resolution at a cellular level..$^{15,40}$ Consequently, individual neoplastic cells can escape detection and evade resection. Acquiring real-time microscopic information during tumor resection could increase the extent of resection and improve patient survival.

Rapid intraoperative diagnosis is important for distinguishing between neoplasms requiring different surgical paradigms (for example, lymphomas vs gliomas) or for guiding the extent of resection. "Frozen section" is currently the most rapid intraoperative histopathological method; however, this method lacks interactivity. The sample preparation equipment is located outside the operating room, and a mean interval of about 20 minutes is 
required to analyze one biopsy sample. ${ }^{1,30}$ Sampling error, artifacts, or both can preclude rapid and accurate diagnosis from a frozen section. Consequently, this technique is too impractical to efficiently guide the extent of resection.

Fluorescence imaging techniques have shown great potential for labeling cells and metabolic processes. ${ }^{25,41}$ Intraoperative confocal endomicroscopy has been evaluated extensively with in vivo imaging in the human gastrointestinal system. Gynecological endomicroscopy and laparoscopy have significantly improved the diagnostic yield of biopsies. . $^{13,39,44-46}$ A miniaturized confocal laser endomicroscope integrated into the distal tip of a conventional video-endoscope now allows confocal fluorescent imaging of brain tissue in vivo. . $2,25,35-37^{-1}$

We recently investigated the feasibility of two handheld portable fiberoptic confocal endomicroscopes for acquiring in vivo images of experimental gliomas using fluorescent dyes. A 488-nm (Optiscan 5.1) visible-wavelength confocal endomicroscope (VWCE) system using fluorescein sodium (FNa) and acriflavine hydrochloride (AF) and a 780-nm near-infrared confocal endomicroscope (NIRCE) system (both from Optiscan Pty., Ltd.) using indocyanine green (ICG) were used in a murine malignant glioma model to distinguish among normal brain, microvasculature, and tumor margins. ${ }^{25,37} \mathrm{We}$ have also assessed the feasibility of using the VWCE with FNa in human brain tumor surgery and explored its use with 5-aminolevulinic acid (5-ALA) in gliomas. Currently, 5-ALA is not approved for intracranial applications in the US, although it is approved to aid malignant glioma resection in Europe and Asia. ${ }^{12,35}$ From our recent studies it is clear that such portable confocal imaging systems can provide rapid screening of multiple observational fields of tissue.

Many fluorophores are used with benchtop confocal microscopes that utilize various ranges of laser excitation wavelengths. However, identifying which fluorophores are compatible, functional, and efficacious with the more limited capabilities of a portable miniaturized laser confocal system is crucial for defining the surgical utility of the system. Conceptually, portable interactive microscopic imaging in human brain surgery would seem to be advantageous. However, in vivo application of this technology in humans, for example in gastroenterology or dermatology, is currently limited to a relatively small number of clinically available fluorophores. Even fewer fluorophores are approved for in vivo neurosurgical applications ( $\mathrm{FNa}$, 5-ALA, and ICG). For our first work involving a murine glioblastoma model, in vivo fluorescent dyes were limited to $\mathrm{FNa}$ and $\mathrm{AF}$, and the images were relatively low resolution. ${ }^{37}$ However, other rapid-acting fluorescent dyes can be used on tissue samples in vivo and ex vivo to reveal more detailed histopathological features.

Since our previous study, ${ }^{37}$ the Optiscan 5.1 VWCE system has been modified and upgraded to provide higherresolution images with an improved signal-to-noise ratio. The Optiscan 5.1 system has set a benchmark as the first clinical portable VWCE system for use in neurosurgery. Therefore, we sought to expand, refine, and define the imaging results and utility of the currently available VWCE system. We used both nonspecific (FNa, acridine orange [AO], AF, cresyl violet [CV], and 5-ALA) and more spe- cific fluorophores (sulforhodamine 101 [SR101]), all of which have imminent potential for in vivo or ex vivo use for defining histopathological features of relevant experimental rodent malignant gliomas. Another goal of this study was to describe the normal brain cytoarchitecture of a large animal since such controlled use in defining normal brain regions and structures has not been performed with the VWCE system.

\section{Methods}

Ethics Approval

All animal investigations were performed according to the guidelines outlined by the National Institutes of Health Guide for the Care and Use of Laboratory Animals after obtaining approval from the Institutional Animal Care and Use Committee of the Barrow Neurological Institute and St. Joseph's Hospital and Medical Center, Phoenix, Arizona. Animals were maintained in the vivarium of St. Joseph's Hospital and Medical Center.

\section{Intracranial Implantation of Murine Glioma}

Ten-week-old female B6 (Cg)-Tyr ${ }^{\mathrm{c}-2 \mathrm{~J} / \mathrm{J}}$ (albino variant of C57BL/6) mice (25 animals) weighing an average of $20 \mathrm{~g}$ were anesthetized and placed in a small-animal stereotactic headframe. The GL261 tumor cells (Division of Cancer Treatment and Diagnosis, National Cancer Institute) were infused at a depth of $2.5 \mathrm{~mm}$ from the brain surface through a bur hole at the targeted brain location after the syringe needle was withdrawn $0.5 \mathrm{~mm}$ to a total depth of $2.0 \mathrm{~mm}$ below the surface of the brain to create a $0.5-\mathrm{mm}$ pocket for the cells. The $2-\mu \mathrm{l}$ cell suspension was infused using a controllable microinjector $(0.67 \mu \mathrm{l} /$ min $\times 3$ minutes with the needle in place for 2 minutes afterward).

\section{Intracranial Implantation of Rat Glioma}

Five-week-old Crl:NIH-Foxn1 ${ }^{\text {rnu }}$ rats (5 animals) were anesthetized and placed in a stereotactic headframe. The U251 glioma cells (American Type Culture Collection) were infused at a depth of $4.5 \mathrm{~mm}$ below the brain surface into the caudate putamen after the syringe needle was advanced $5.0 \mathrm{~mm}$ to create a $0.5-\mathrm{mm}$ pocket for the cells. The $10-\mu l$ cell suspension was infused using a controllable microinjector $(3.33 \mu \mathrm{l} / \mathrm{min} \times 3$ minutes with the needle in place for 2 minutes afterward).

\section{Animal Surgery}

Twenty-one days after tumor cell implantation, the mice and rats underwent craniectomy. An operating microscope (Zeiss Pentero, Carl Zeiss Surgical GmbH) and intraoperative confocal imaging were used to visualize exposed surfaces of both cerebral hemispheres. The animals were anesthetized, and their oxygen supply and body temperature were maintained throughout the procedure. Intraparenchymal brain tumors were identified macroscopically in the right hemisphere of all mice and rats. After imaging was performed, the anesthetized animals were killed according to guidelines.

\section{In Vivo Imaging Using the VWCE}

The VWCE system incorporates a handheld minia- 


\section{Handheld confocal microscopy with various rapid fluorescent dyes}

turized scanner containing a rigid probe with a $6.3-\mathrm{mm}$ outer diameter, providing a working length of $150 \mathrm{~mm}$. A 488-nm diode laser provided incident excitation light. Fluorescent emission was detected at 505-750 nm for 5-ALA and SR101 and at 505-585 $\mathrm{nm}$ for the remaining fluorophores using a band-pass filter, via a single optical fiber acting as both the excitation and detection pinholes for confocal isolation of the focal plane. The detector signal was digitized synchronously with the scanning to construct images parallel to the tissue surface (en face optical sections). Laser power was typically set to 550-900 $\mu \mathrm{W}$ for brain tissue; maximum power was limited to $1000 \mu \mathrm{W}$. An FOV of $475 \times 475 \mu \mathrm{m}$ (magnification approximately 1000 on a 21 -inch screen) was scanned at $1024 \times 512$ pixels $(0.8 / \mathrm{sec}$ nd frame rate) or at $1024 \times 1024$ pixels $(1.2 /$ second frame rate) with a lateral resolution of $0.7 \mu \mathrm{m}$ and an axial resolution (that is, effective optical slice thickness) of approximately $4.5 \mu \mathrm{m}$. The resulting images were stored digitally and could be recorded as time-lapse series.

During the procedure, a foot pedal provided remote control of the variable confocal imaging plane depth from the surface to a depth $0-500 \mu \mathrm{m}$. The Optiscan 5.1 instrument is unable to image through thick objects (for example, skull), although it can obtain images through the arachnoid membrane and cells up to $500 \mu \mathrm{m}$. The confocal probe affixed to a retractor system was moved gently along the surface of the brain tissue to obtain images brain, regions of obvious tumor, and the transitional zone between what appeared to be normal brain and tumor. The probe was moved smoothly between different ROIs without losing contact with the tissue. Unique aspects of individual cells and surrounding neoplastic tissue were seen as the VWCE probe acquired images throughout its focal depth range. The total imaging time was 20 minutes per animal for tumor models. Total imaging time for the porcine anatomical investigations was 2 hours for each animal.

In addition to the in vivo experiments, we harvested tissue samples with a no. 11 scalpel blade, immediately stained them, and imaged them ex vivo with the VWCE and laser scanning microscope (LSM; LSM710, Carl Zeiss $\mathrm{GmbH}$ ). To compare the handheld endomicroscope images with the images collected under optimal benchtop confocal parameters, we equipped the LSM with an $\times 40 / 1.2$ NA objective, set the confocal aperture for one Airy unit, and sampled at Nyquist.

\section{Fluorophores}

We selected rapid-staining fluorophores that included agents approved for clinical use in humans (including for use in brain and non-brain tissue). As much as possible, concentrations were kept consistent with dosages used in humans. Properties of the fluorophores and routes of administration for imaging murine gliomas are summarized in Table 1.

In vivo imaging using VWCE was performed 5 minutes after administration for FNa (5 animals), immediately for AF (5 animals) and AO (5 animals), 10 minutes after administration for CV (5 animals), and 2 hours after administration for 5-ALA (5 animals). To investigate VWCE imaging using combined sequential fluorescent from several regions of interest (ROIs), including normal

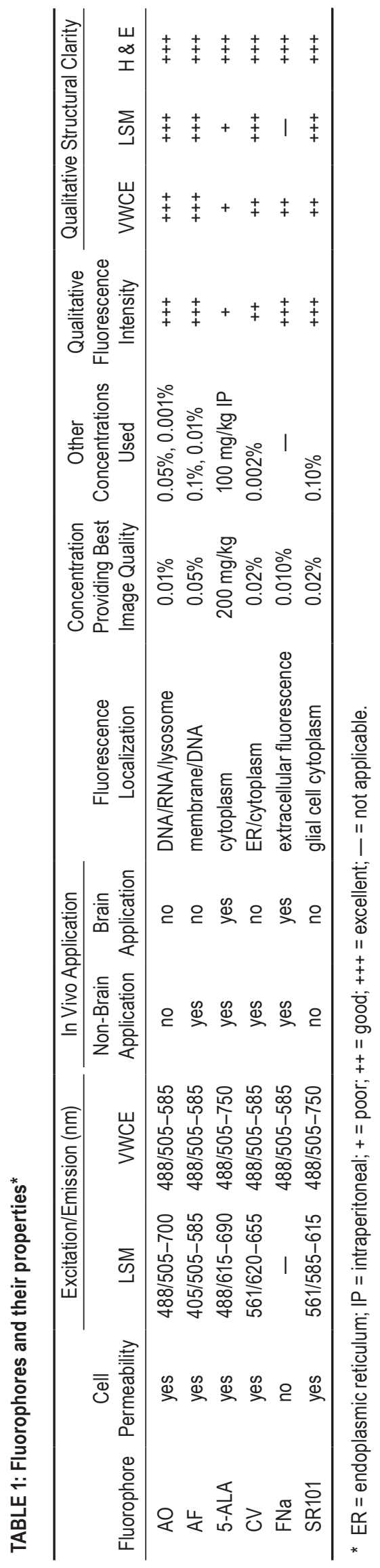

Neurosurg Focus / Volume 36 / February 2014 
dyes, AO was topically applied to the tumor after injecting the animal with FNa. Visible-wavelength confocal endomicroscope imaging was also performed on ex vivo tissue stained with $\mathrm{AO}, \mathrm{AF}$, and $\mathrm{CV}$.

Animals injected with 5-ALA were imaged either in vivo with the VWCE or ex vivo with the LSM using a diode-pumped, solid-state 488-nm laser coupled with a 615- to 690-nm band-pass filter. Rapid bleaching and weak fluorescence of protoporphyrin IX (PpIX) did not allow the same samples to be imaged with both the handheld and the benchtop confocal systems. During ex vivo imaging, the laser and gain were adjusted to fill the dynamic range of the detector. The confocal aperture was set to minimize detection of fluorescence from outside the focal plane. To differentiate the fluorescence contributions of PpIX from autofluorescence, emission spectra were sequentially collected from identical regions with a 32-channel meta-analyzer incorporating the same 488-nm laser excitation. Data were collected from regions exposed to less than 10 seconds of combined bright field and laser excitation to minimize photobleaching.

SR101 (5 animals) was topically administered (250$\mu$ mol concentration; human dosages not yet determined $)^{27}$ to exposed rat brain and tumor tissue. A separate group (5 animals) received an intracarotid injection of SR101 (1 ml of 250- $\mu \mathrm{mol}$ solution). One hour after SR101 exposure, the tissue was rinsed with artificial CSF and imaged in vivo with VWCE, followed by scalpel excision of the same tissue region and immediate ex vivo imaging of the same tissue with LSM and conventional histological examination after $\mathrm{H} \& \mathrm{E}$ and glial fibrillary acidic protein (GFAP) antibody staining.

\section{Tissue Sampling and Correlative Histology}

Brain and tumor areas imaged in vivo using the VWCE were marked with tissue ink so that locations could be validated precisely for LSM imaging and correlated as exactly as possible with $\mathrm{H} \& \mathrm{E}$ and GFAP histology. The tissue was placed in a cassette for standard formalin fixation and paraffin embedding. Histological assessment was performed via standard light microscopy evaluation of 5- $\mu$ m-thick, $\mathrm{H} \& \mathrm{E}$-stained sections using an Olympus BX60 upright microscope.

\section{Normal Brain Imaging Using VWCE}

Three adult Yorkshire swine were used to investigate the utility of the VWCE to image features of larger, normal brains. The animals were anesthetized, and their oxygen supply and body temperature were maintained in homeostasis throughout the procedure. Craniectomy and C1-5 laminectomy were performed on each anesthetized animal. The brain and upper cervical spinal cord were excised with the surrounding dura mater. After these procedures the anesthetized animals were killed according to guidelines. Using a tissue slicer, we cut 5-mm-thick slices and soaked them in $0.01 \%$ AO for 2 minutes and imaged them with the VWCE. Imaged anatomical areas were carefully documented and marked with tissue ink, as previously described, to correlate $\mathrm{H} \& \mathrm{E}$-stained sections with confocal images.

\section{Results}

\section{Imaging of Rodent Glioma With the VWCE and LSM}

Fluorescein Sodium. Confocal endomicroscopy with the VWCE revealed intravascular distribution in the normal brain within 5 minutes after FNa injection. Arterioles and capillaries contrasted sharply against the weakly fluorescent background of normal brain tissue. Tumor areas with disrupted blood-brain barrier (BBB) were characterized by extravasation of FNa. Typical histopathological features such as pleomorphism, atypia, and hypercellularity contrasted against the fluorescent extracellular background. Mitoses were suspected in some cells, but the imaging was not clear enough to confirm their presence. Cells appeared as dark silhouettes; moreover, intracellular components appeared as shadows of varying grayscale intensities. The FNa fluorescence intensity dynamics across tumor cells showed delineation of nuclei, which was confirmed with subsequent $\mathrm{AO}$ counterstaining. Nuclei appeared darker than cytoplasm and could be defined in most cases. Tumor boundaries could be observed based on cellularity. Extracellular matrix and tissue architectural features were difficult to distinguish from blood and cells. Erythrocytes appeared as multiple, round, regularshaped cells visually smaller than tumor cells. Pooling of erythrocytes within the imaging field produced artifacts that decreased image quality and affected analysis. In some areas, blood artifacts, especially erythrocyte conglomerates, impeded delineation of tumor boundaries and required saline irrigation of the tissue field, cleaning of the VWCE objective, or both (Fig. 1).

Acridine Orange and AF. Acriflavine hydrochloride and $\mathrm{AO}$ stained tissue rapidly and immediately. Both dyes reveal excellent nuclear detail and weak cytoplasmic staining of tumor cells, providing contrast among the nuclei, cytoplasm, and extracellular space. Structures appear intensely fluorescent over a dark background. Normal brain appears as hypocellular tissue with uniformly shaped cells, while tumor cells tend to be brightly delineated. Endothelial cells lining vasculature are also visualized with bright nuclei. Typical glioma cytoarchitecture reveals hypercellularity, atypia, and mitotic figures. Tumor boundaries are easily differentiated from adjacent normal brain based on cellularity (Figs. 2 and 3). Unlike AO, which predominantly stains nuclei, AF provides some cell membrane contrast. This feature allows observation of more cell volume with AF than with AO. The AO, which is predominantly a nuclear stain within tumor cells, provides sharper delineation of mitoses and shapes of intranuclear components. Systemic administration of $\mathrm{AO}$ and $\mathrm{AF}$ at a concentration sufficient for fluorescence imaging $(0.5 \mathrm{ml}$ of $0.1 \% \mathrm{AF}$ and $0.01 \% \mathrm{AO}$ ) was lethal for the animals.

In most cases, in vivo and ex vivo imaging with the VWCE provided similar histological information. Ex vivo imaging with an LSM produced higher-resolution images than the VWCE and optimally coupled excitation and emission spectra. No blood artifacts appeared on any ex vivo images; however, the LSM identified the same histological features as the VWCE with AO and AF.

Topical application of $0.1 \% \mathrm{AO}$ after systemic injec- 

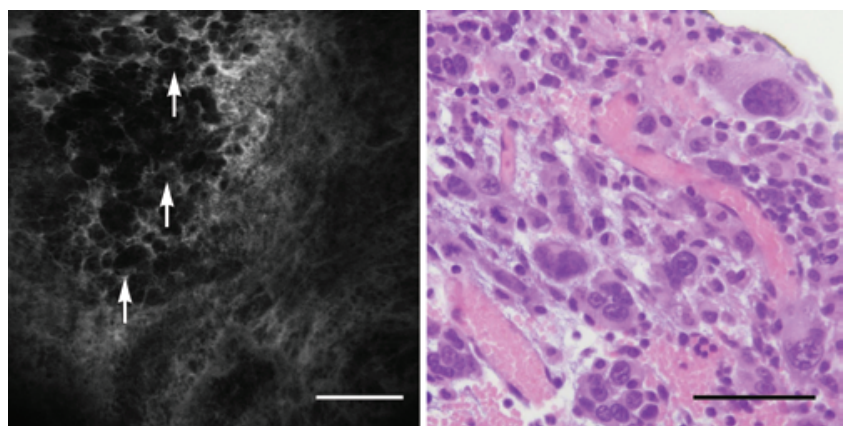

FIG. 1. Left: In vivo image of the tumor obtained with a VWCE, demonstrating extracellular distribution of FNa. Atypical cells (arrows) consistent with tumor cells appear within a cellular mass. Printed with permission from Barrow Neurological Institute. Right: Photomicrograph from the same ROI demonstrates atypical cells within the tumor, similar to the in vivo image. $\mathrm{H} \& \mathrm{E}$. Bar $=100 \mu \mathrm{m}$.

tion of FNa immediately "supplants" FNa. Extracellular background fluorescence of FNa converted to characteristic AO fluorescence with intracellular labeling. No extracellular fluorescence, typical for FNa, was observed (Fig. 4).

Cresyl Violet. Cresyl violet acetate is a rapid stain with an optimal incubation time of 5 minutes. It is predominantly localized to the cytoplasm with minimal staining of nuclei and extracellular space. Dark nonflourescent silhouettes, consistent with nuclei, were sharply delineated by bright fluorescent cytoplasm. The nuclear/cytoplasmic ratio could be assessed. The cytoplasm, sharply outlined by bright fluorescence, provides information about cell shape. Fluorescent patterns within tumor areas revealed atypia, mitosis, and pleomorphism. Within normal brain, fluorescence of white matter was minimal and staining of cellular structures (for example, ependyma of lateral ventricles) was strong. Architectural characteristics of white matter were not seen well because of dim grayscale fluorescence, which was similar to the intensity of background fluorescence. Histological features could not be distinguished. Although imaging of cellular structures revealed fluorescent cytoplasmic labeling, fluorescence intensity with CV tended to be dim. Consequently, the tumor-normal brain interface was difficult to visualize because of the low signal-to-noise ratio (Fig. 5).
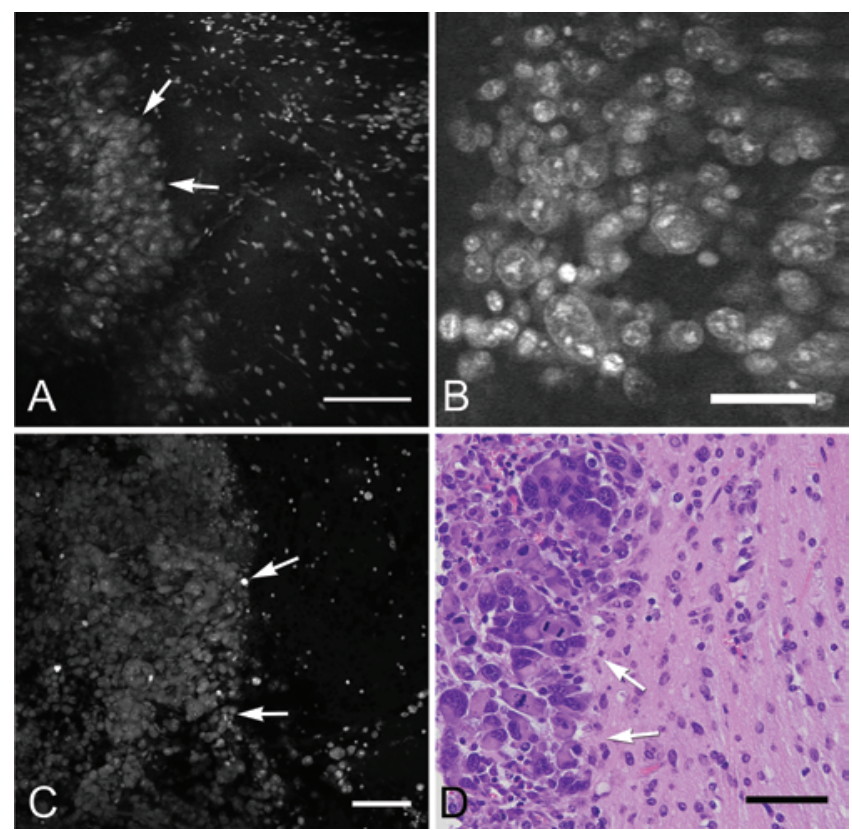

Fig. 3. Images obtained from tumor border. In vivo image obtained with a VWCE (A), demonstrating tumor cells stained with AO (arrows). Magnified VWCE image (B) shows detailed intracellular components of tumor cells. Ex vivo AO-stained image obtained with an LSM (C) and photomicrograph (D) of $\mathrm{H} \&$ E-stained tissue from the same ROI show comparable histological features (arrows). Bar $=100 \mu \mathrm{m}$. Images labeled $\mathrm{A}-\mathrm{C}$ printed with permission from Barrow Neurological Institute.

Imaging of CV with the LSM revealed typical fluorescent patterns in normal brain. Normal brain appeared as brighter-than-background fluorescent architecture with multiple regular-shaped dark shadows of nuclei. The better results using benchtop confocal microscopy imaging with CV could be explained by optimally coupled excitation and emission spectra.

5-Aminolevulinic Acid. Two hours after an injection of 5-ALA, we identified PpIX fluorescence from individual cells within tumor regions by using the VWCE. Unlike other fluorophores, 5-ALA did not provide histological information when compared with $\mathrm{H} \& \mathrm{E}$ and appeared as clusters of bright pixels. Cell outlines, intracellular structures, or extracellular matrix were not distinguishable
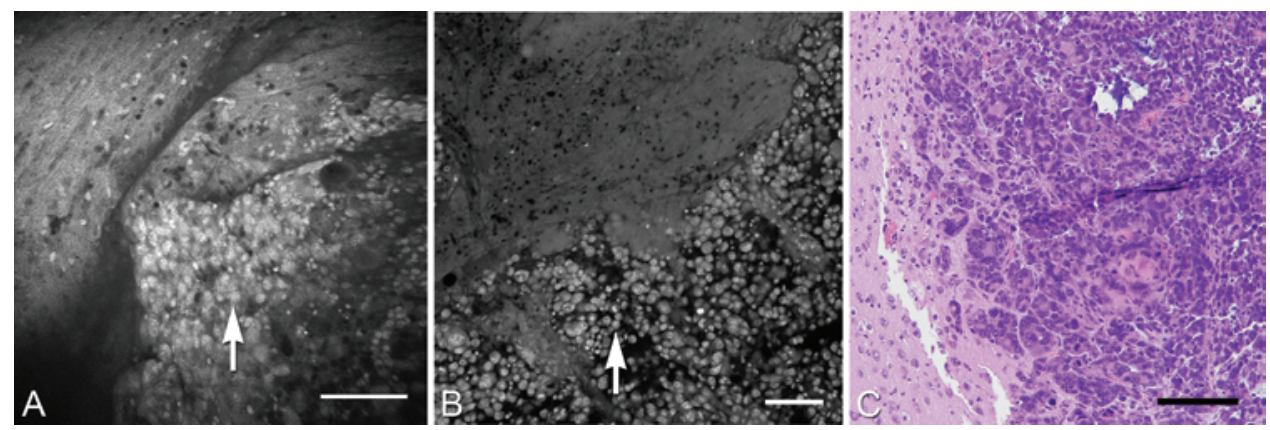

FIG. 2. In vivo image obtained with a VWCE (A) at the tumor border, demonstrating intracellular distribution of AF. Atypical cells (arrows) consistent with tumor cells appear within regions of increased cellularity and infiltrating edge. Ex vivo AF-stained image obtained with LSM (B). Photomicrograph (C) of $\mathrm{H} \&$ E-stained tissue from the same ROI featured in panel A demonstrating atypical cells within the tumor and tumor edge, similar to the in vivo image. Bar $=100 \mu \mathrm{m}$. Images labeled $A$ and $B$ printed with permission from Barrow Neurological Institute. 

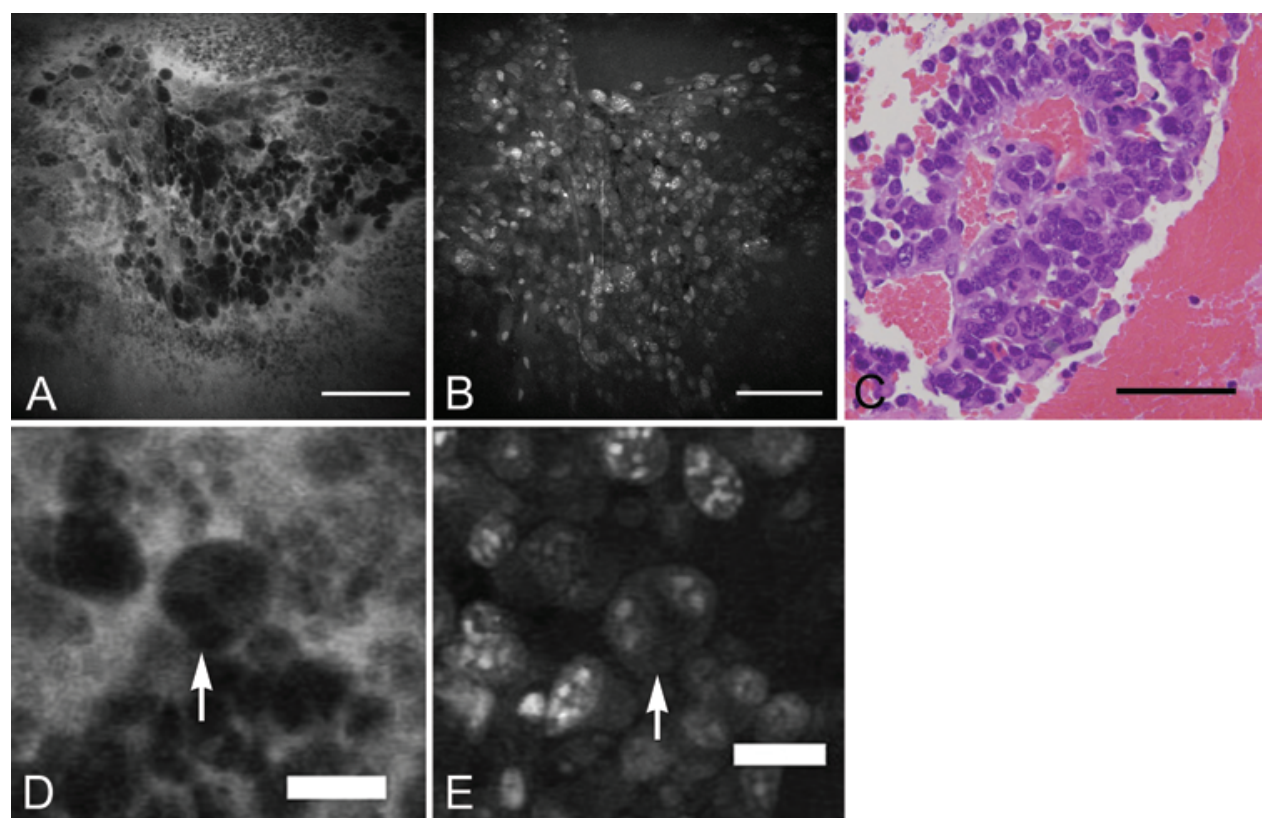

FIG. 4. In vivo images obtained with a VWCE, demonstrating a tumor region after FNa injection (A) and the same field of view after AO injection (B) showing immediate "supplanting" of FNa fluorescence. Photomicrograph (C) from same region showing similar characteristics of tumor (atypia, pleomorphism). H \& E. Bar $=100 \mu \mathrm{m}$. Magnified FNa-stained (D) and AO-stained (E) confocal images reveal a possible mitotic figure in a same tumor cell (arrows). Bar $=50 \mu \mathrm{m}$. Images labeled A, B, D, and E printed with permission from Barrow Neurological Institute.

from each other. Normal brain areas showed a minimal amount of fluorescent pixels over background noise.

Protoporphyrin IX fluorescence detected in tumor regions with the LSM delineated individual cells. Spectral imaging of these cells verified an emission peak distinct from PpIX at $635 \mathrm{~nm}$. This finding differed from findings in normal tissue, in which the minimal fluorescence detected beyond $620 \mathrm{~nm}$ could not define cells morphologically. We also identified pixels within normal tissue containing emission peaks at $635 \mathrm{~nm}$. These individual pixels could not delineate PpIX-positive cells.

Protoporphyrin IX fluorescence appeared bright during standard widefield visualization. However, the fluorescence signal was attenuated when confined to a narrow focal plane by the LSM. As in previous reports, we ob- served rapid photobleaching of PpIX in cells during light excitation (Fig. 6).

Sulforhodamine 101. Sulforhodamine 101 provides bright fluorescence after 60 minutes of topical application. Cytoplasmic staining with SR101 was easily distinguished from low background fluorescence using the VWCE. In some cells intracellular components could be identified as dark nonfluorescent silhouettes within the bright fluorescent cell. Pleomorphic cells in frank tumor regions were brightly fluorescent. Within normal brain tissue, fluorescent cells with extensive processes, likely astrocytes, could be identified. Tumor tissue was labeled much more intensely than normal brain tissue (Fig. 7). Architectural characteristics of nontumorous brain tissue were not seen well.
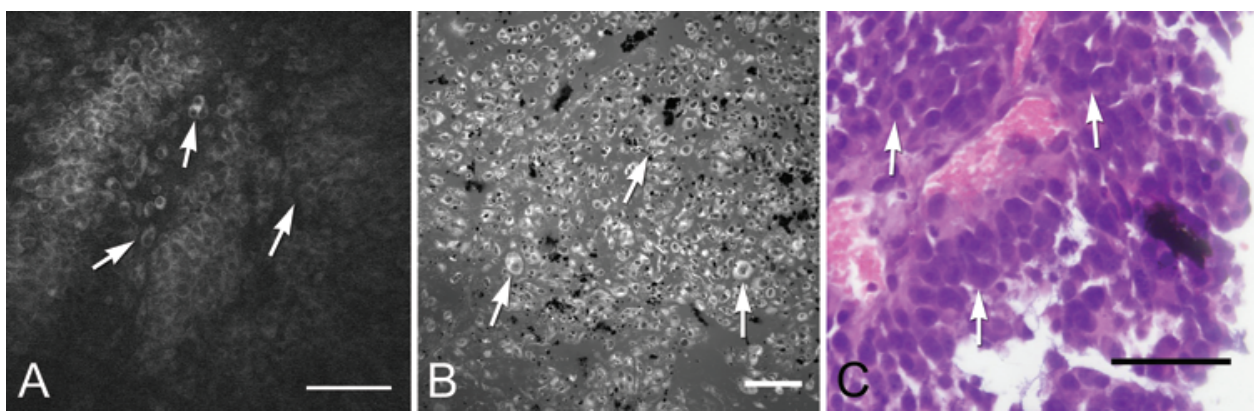

FIG. 5. Images obtained from tumor area. In vivo CV-stained image obtained with a VWCE (A), demonstrating atypical tumor cells (arrows). Ex vivo CV-stained image obtained with an LSM (B) and H \& E-stained tissue photomicrograph (C) from the same $\mathrm{ROI}$, showing similar histological features (arrows). More optimized excitation-emission coupling in the LSM improves image quality for CV, because of the laser limitation of the VWCE for imaging CV at $488 \mathrm{~nm}$. Bar $=100 \mu \mathrm{m}$. Images labeled A and B printed with permission from Barrow Neurological Institute. 

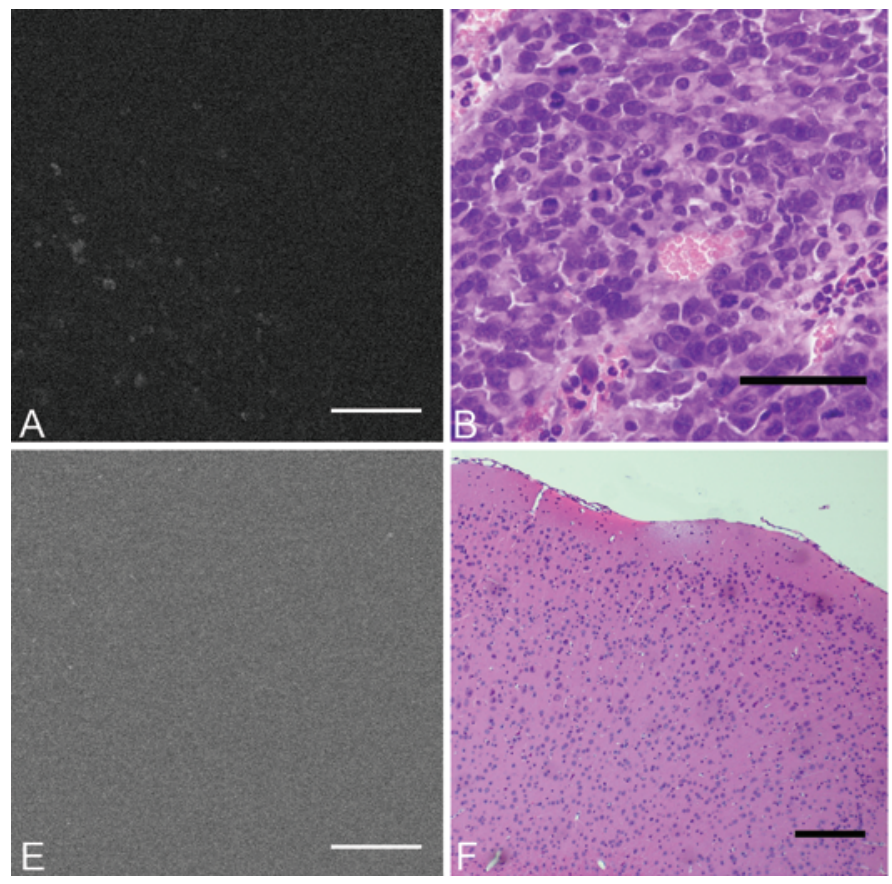
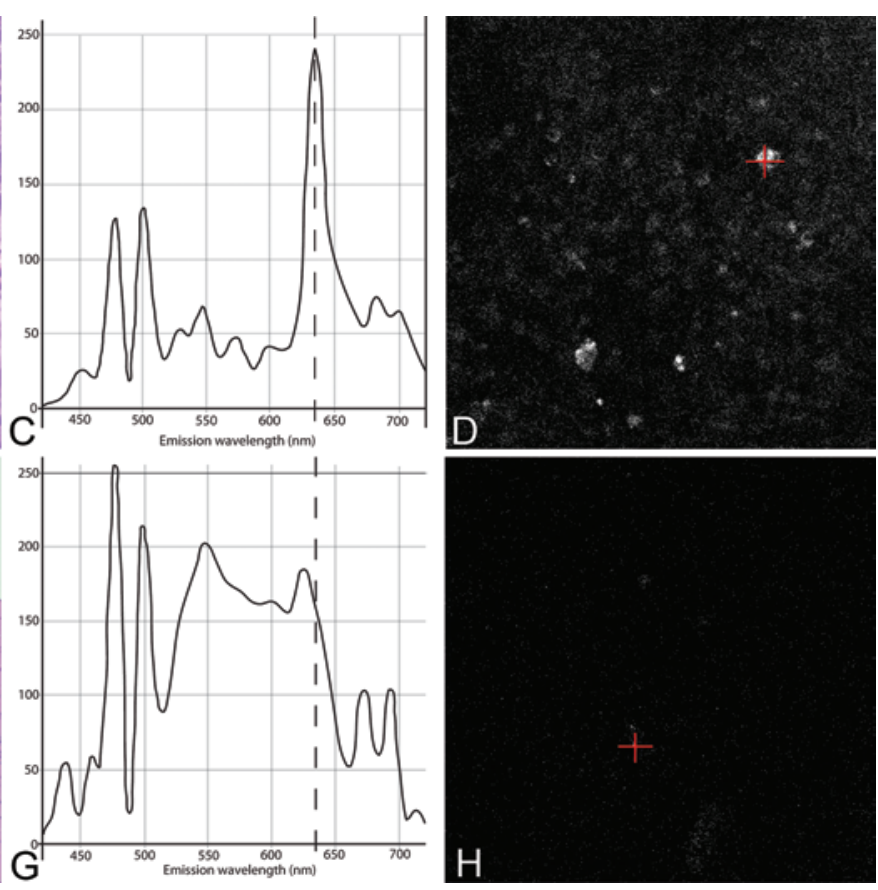

FIG. 6. A VWCE image (A) with 5-ALA obtained from the tumor area, highlighting clusters of indistinct pixels. A photomicrograph (B) of $\mathrm{H} \& \mathrm{E}$-stained tissue from the same ROI shows tumor clearly. Spectral imaging of cells (C) identified with an LSM verified an emission peak at $635 \mathrm{~nm}$ for PpIX, distinct from fluorescent cells in tumor area (D, red cross). A VWCE image (E) from normal brain tissue region revealed scattered minimal fluorescence, whereas $\mathrm{H}$ \& E-stained tissue (F) from the same ROI confirms normal brain architecture. Spectral imaging $(\mathbf{G})$ of fluorescent pixels $(\mathbf{H}$, red cross) in normal brain tissue imaged with LSM detected minimal fluorescence beyond $620 \mathrm{~nm}$. Bar $=100 \mu \mathrm{m}$. Images labeled A, D, E, and H printed with permission from Barrow Neurological Institute.

\section{Ex Vivo Imaging of Normal Porcine Brain and Spinal Cord Using a VWCE}

Visible-wavelength confocal endoscopic imaging of normal porcine brain revealed distinct histological information that corresponded to findings on $\mathrm{H} \& \mathrm{E}$ staining of different anatomical regions. Frontal cortex was characterized by dark neuron cell bodies surrounded by brighter and smaller cells, possibly glial cells. Based on shape and their course, bright channels could be interpreted as blood vessels. Imaging of frontal white matter revealed axons and multiple bright fluorescent cells, which were possibly glial cells. A few cross-sectioned vessel lumina were seen within the field of view as bright fluorescent oval-shaped structures and darker perivascular spaces. The caudate nucleus consisted of dark white matter tracts and neuron cell bodies over a brighter background. The walls of the lateral ventricle showed a characteristic honeycomb pattern of intensely fluorescent ependymal cells. Prominent crossing fibers were evident in the pons. Imaging of the medulla revealed crossing fiber tracts in different planes. In the cerebellum, Purkinje cells appeared as large dark cells. These cells were identified adjacent to the granular cell layer from one side, which appeared as hypercellular and intensely fluorescent. White matter tracts surrounded Purkinje cells from the opposite side. The gray matter of the spinal cord consisted of large, dark neuron cell bodies. Images from the central canal were outlined with intensely fluorescent ependymal cells. White matter appeared as cross-sectioned myelinated axons (Fig. 8).

\section{Discussion}

In this study we describe an expanded application of intraoperative handheld confocal endomicroscopy at 488 $\mathrm{nm}$ using the Optiscan 5.1 VWCE for imaging animal models of malignant glioma and normal brain and spinal cord tissue with a variety of rapid fluorescent dyes. The technique enabled straightforward identification of the same histological features of tumor and normal brain as those seen with standard benchtop confocal microscopy and conventional $\mathrm{H} \& \mathrm{E}$ staining. Imaging of $\mathrm{AO}$-stained normal brain slides provided characteristic histological findings from corresponding anatomical areas correlating to $\mathrm{H} \& \mathrm{E}$ sections. The results suggest that in vivo VWCE can provide real-time histological information. Such information could facilitate differentiation of tumorcontaining areas and cytoarchitecture from normal brain tissue depending on the action of the fluorescent agent. Furthermore, VWCE imaging after fluorescent staining provided refined, high-resolution images that offered precise ex vivo histology within 1-5 minutes of tissue sampling.

\section{Portability of the Imaging System}

An obvious advantage of such handheld imaging technology is its portability. The system can be easily moved from one operating room to another. The profile of the laser probe can easily fit within the instrument working area of the neurosurgical operating microscope. The system can also be used to instantly transmit acquired 

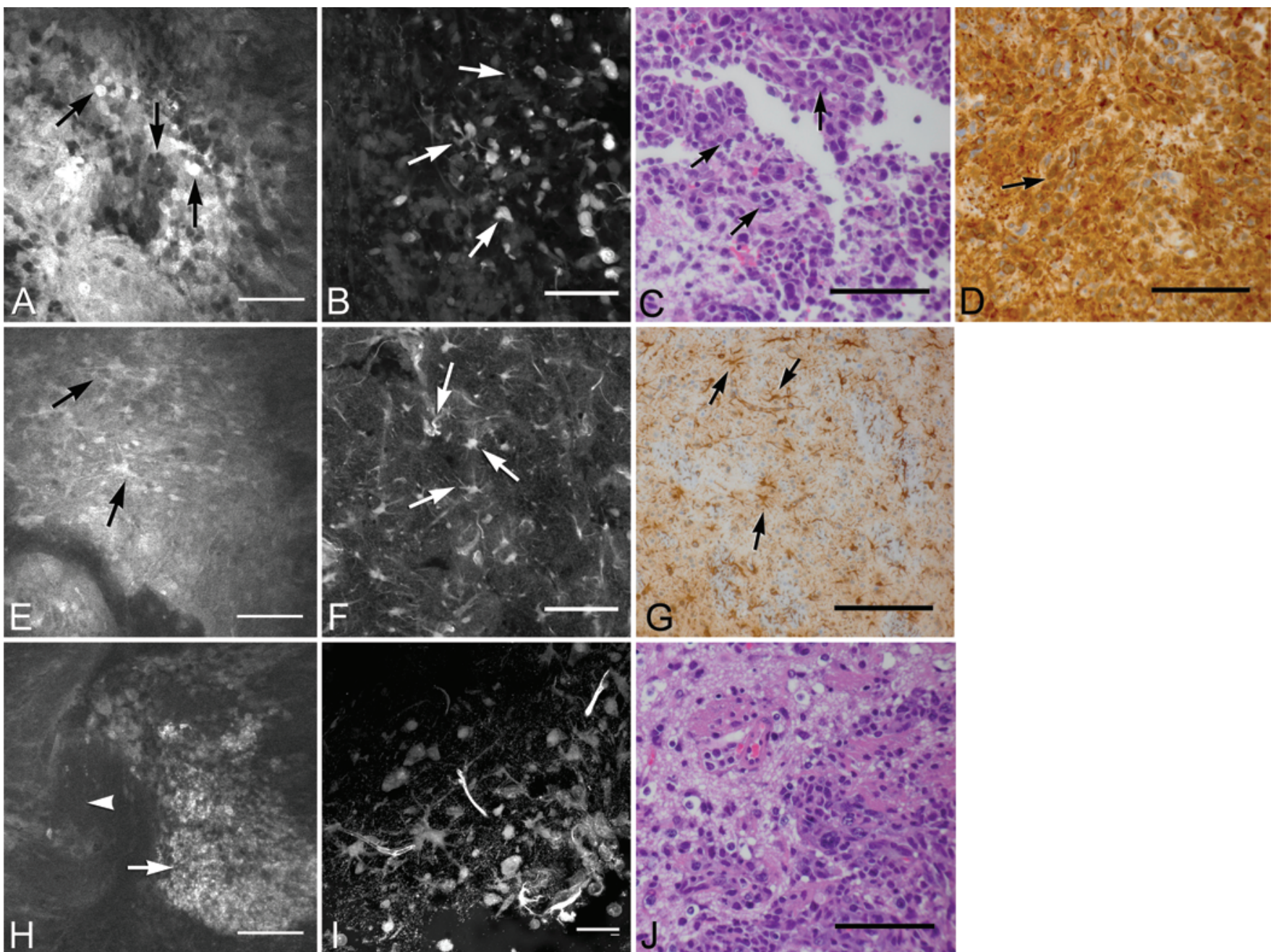

FIG. 7. In vivo SR101-stained image obtained with a VWCE (A), demonstrating atypical tumor cells within a tumor mass (arrows). Ex vivo image obtained with an LSM (B) and H \& E- (C) and GFAP-stained (D) tissues from the same ROI show similar histological features (arrows). In vivo image obtained with a VWCE (E) and an ex vivo image obtained with an LSM (F) highlight reactive astrocytes (arrows) within the peritumoral area. Glial fibrillary acidic protein-stained tissue (G) from the same ROI confirms reactive astrocytes (arrows). In vivo image obtained with a VWCE (H) and an ex vivo image obtained with an LSM (I) at the tumor border, showing a hypercellular tumor area (arrows) and adjacent edematous brain parenchyma (arrowheads). Compare with the $\mathrm{H}$ \& E-stained tissue $(\mathrm{J})$ from the same $\mathrm{ROI}$ revealing similar histological features. Bar $=100 \mu \mathrm{m}$. Images labeled A, B, $E, F, H$, and I printed with permission from Barrow Neurological Institute.

images without waiting for digital images of conventional histological slide preparations from biopsies. Such technology could hasten the transmission of the pathologist's analysis to the neurosurgeon..$^{12}$

The ability of intraoperative in vivo imaging with miniaturized ergonomic probes to acquire microscopic images over multiple fields of view more rapidly than frozen-section analysis holds great potential. Importantly, however, there are differences in what to expect from relatively nonspecific fluorescent staining compared with more conventional histopathological techniques. Exact cellular characterization may not be necessary to achieve usefulness and practicality in a surgical setting. The use of approved fluorescent dyes to image, interpret, and confirm general cytoarchitectural features may allow identification of tumor, tumor border, and normal brain, thus influencing the neurosurgical procedure.

\section{Limitations of Fluorophores and Imaging Parameters}

A limited number of fluorescent agents are approved for clinical in vivo use in the human brain, including $\mathrm{FNa}$, 5-ALA, and ICG. Fluorophores such as AO, AF, CV, SR101, although not approved for in vivo brain use but approved for other organ system use, were used in this study because they may find some in vivo brain use with modifications and because they were originally used in the development of the Optiscan 5.1 VWCE. Our previous laboratory experience with the VWCE and NIRCE using ICG showed that this technique can reveal tumor regions, boundaries, and normal brain. After systemic administration of ICG, fluorescence was time-dependent, with optimal fluorescence occurring 15 minutes after ICG administration. However, topical administration of ICG produced near-immediate fluorescent staining. In com- 

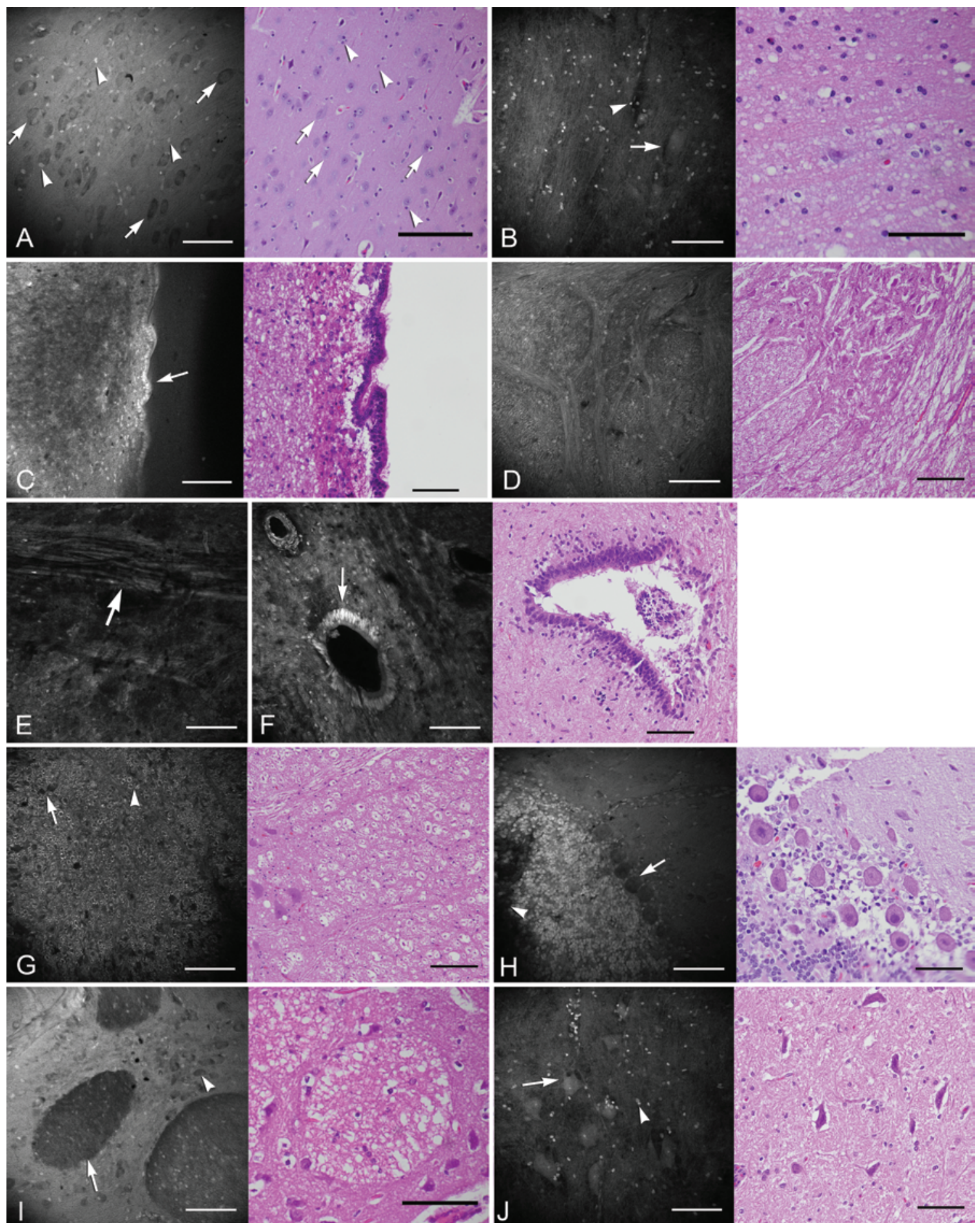

FIG. 8. Normal brain stained with $A O$ and imaged with a VWCE (left) compared with corresponding $\mathrm{H}$ \& E-stained tissues from the same ROI (right). Confocal image (A) from frontal cortex highlighted by neuron cell bodies (arrows) surrounded by glial cells (arrowheads). Image (B) from frontal white matter reveals axons (arrow) of neurons and multiple glial cells (arrowhead). Image (C) from the wall of lateral ventricle shows intensely fluorescent ependymal cells (arrow). Image (D) of medulla reveals fiber tracts crossing in different planes. Image (E) of pons highlights prominent crossing fibers (arrow). Image (F) shows the spinal cord central canal outlined with intensely fluorescent ependymal cells (arrow). Image (G) of gray matter of spinal cord revealed dark, large neuron cell bodies (arrow). White matter appeared as cross-sections of myelinated axons (arrowhead). Image (H) from cerebellum highlights Purkinje cells (arrow). White matter tracts and granular cell layer are visible (arrowhead). Image (I) from caudate nucleus demonstrates white matter tracts (arrow) and neuron cell bodies (arrowhead). Image ( $\mathrm{J}$ ) from basal ganglia shows neuron cell bodies (arrow) surrounded by glial cells (arrowhead). Bar $=100 \mathrm{um}$. Left images labeled A-D and F-J and the image labeled E printed with permission from Barrow Neurological Institute. 
parison, $\mathrm{FNa}$ exhibited sufficient fluorescence within 5 minutes after administration. ${ }^{25}$ Although NIRCE technology has the advantage of nearly twice the depth of imaging offered by VWCE, the VWCE technology is closest to realizing a neurosurgical application. The VWCE system was developed first and has been used extensively to increase diagnostic yield of biopsies during gastrointestinal endoscopy using AO, AF, CV, and other dyes. ${ }^{13,39,44-46}$ Thus, adapting technology proven in gastroenterology to neurological questions seems logical for expanding its use. However, numerous specific fluorophores have been developed in the NIR spectrum. Thus, fluorescent agents in the NIR spectrum may find more widespread clinical use in the future..$^{18,31,34}$

Current handheld endomicroscopy systems can only excite fluorophores at fixed wavelengths (488 and $780 \mathrm{~nm}$ ). The number of emission filters is also limited. Only a limited number of fluorophores can be imaged at optimally coupled excitation and emission spectra. This characteristic greatly limits the number of fluorophores that can be imaged with a handheld confocal microscope such as the VWCE or NIRCE system. In contrast, full-scale benchtop confocal systems, such as the LSM, contain multiple laser lines and tunable emission filters. Another drawback of current handheld technology is its significantly less laser power compared with benchtop units.

Further development of single-photon technology such as the VWCE should include increasing its power, resolution, probe ergonomics, and available laser lines in one portable device. Adaptation and miniaturization of other laser scanning techniques, such as two-photon laser scanning microscopy, which is based on the technology of the absorption of two synchronized infrared photons in a confined tissue volume with very short laser pulses, may also lead to applications in human brain tumor imaging. Nonlinear two-photon fluorescent imaging has a better signal-to-noise ratio because fluorescence is excluded outside the focal point, tissue penetration is deeper, and phototoxicity is decreased. ${ }^{10,49}$ Improved power and expanded laser lines of a system like the endomicroscope, and similar to two-photon fluorescence imaging, would allow imaging of inherent tissue fluorescence, reflectance, or autofluorescence in molecules such as nicotinamide adenine dinucleotide phosphate, collagen, lipofuscin, and riboflavin-related compounds, obviating the need to apply or inject fluorescent dyes or tags. ${ }^{8,11,21}$ The current laser excitation parameters of the VWCE system is relatively close to the excitation and emission wavelengths of these molecules. This technology remains in its infancy. Fluorescent tags on approved agents or drugs may help to identify tumor subtypes using an instrument such as the VWCE, which is already approved for human use.

\section{Increasing Experience and Clinical Application to Improve Use}

Gaining experience with the current VWCE system for in vivo human images with FNa and 5-ALA, in addition to imaging tissue ex vivo, could provide an excellent battery of rapid fluorescent cytoarchitectural information. Furthermore, systematic collection and retrospective analysis of those images could lead to the creation of a trainable database of confocal microscopic imaging. Even when used in concert with the dyes described in this article, the VWCE should not be considered to replace $\mathrm{H}$ $\&$ E staining. The information provided by these fluorescent dyes is unique and may rapidly provide important labeling data that can augment the information from the various stains used to identify different cellular, subcellular, or extracellular structures in standard histological preparations. In vivo clinical applications with Optiscan 5.1 are limited to a small number of clinically approved fluorophores; however, FNa, ICG, and 5-ALA are among those relatively safe for in vivo human applications. These fluorophores have been routinely used for intraoperative tumor diagnosis at our institution. Furthermore, AO, AF, $\mathrm{CV}$, and SR101, although not approved for human in vivo use, have been used for rapid ex vivo histological diagnosis (work in progress). We have also demonstrated the excellent utility of FNa as an adjunct for clinical histological diagnosis. ${ }^{12}$ The fluorescent dyes under discussion possess unique characteristics in terms of application mode, time for staining, and structures visualized, but using them is no different in principle from using various stains for specific structures in standard histology. The advantage of such portable fluorescent imaging technology is that it offers expanded applications such as colabeling and specific structure localization and identification in vivo or immediately ex vivo.

\section{Fluorophores Approved for In Vivo Human Use}

Fluorescein Sodium. Fluorescein sodium, a nontoxic fluorophore approved by the FDA for retinal angiography, has been used clinically since the 1960s. It has been used extensively for clinical applications in ophthalmology, neurosurgery, and oncology. Its safety profile in ophthalmology is well established. A recent study of 2272 ophthalmological procedures performed at 16 international centers demonstrated that intravenous FNa imaged with laser scanning confocal microscopy caused $1.4 \%$ of patients to suffer mild reactions (for example, nausea, rash), but no one experienced serious adverse events (for example, anaphylaxis). ${ }^{47}$ Fluorescein is one of the oldest fluorophores applied in neurosurgery. It has been used in surgical treatments for arteriovenous malformations, cerebral artery anastomoses, and brain tumor resections. In 1948 FNa was first reported to localize to brain tumors. ${ }^{3,22}$ In this early study FNa was excited with an ultraviolet light source, and "yellow-green" fluorescence was observed in areas of tumor. Low-intensity fluorescence was detected in edematous regions, and healthy tissues did not fluoresce.

Human in vivo use of FNa has been limited to brief periods in the operating room, and our experience with it to image gliomas is limited. In the present study, malignant rodent gliomas provided long, systematic periods for acquiring optimal images and for assessing the cellular characteristics demonstrated by $\mathrm{FNa}$. This fluorophore does not penetrate the normal $\mathrm{BBB}$; thus, confocal imaging of normal brain reveals regional microvascular architecture. In intracranial pathological processes disrupting the BBB, FNa leaks from microvasculature into the extracellular space, providing bright background fluorescence. Cells and extracellular structures appear as shadows over a bright background with different grayscale intensities. 


\section{Handheld confocal microscopy with various rapid fluorescent dyes}

Intracellular structures can be detected for some cells; however, most cells appeared as rounded, homogeneously fluorescent structures. Furthermore, blood artifacts can significantly affect image quality. Nevertheless, given our clinical experience, handheld confocal microscopy with FNa was considered to be useful for real-time in vivo histopathological assessment of a diversity of intracranial lesions. In our animal experiments with a malignant glioma, it appeared that FNa is well suited to show general cytoarchitectural characteristics. Exact discrimination of most cell types may be difficult given that the images produced using FNa revealed staining of the background, and in most cases FNa does not stain cells or intracellular structures themselves. ${ }^{26,37}$

5-Aminolevulinic Acid. This compound is an indirect fluorophore, meaning that it is converted in situ to an activated fluorescent state. After oral administration, 5-ALA endogenously induces PpIX, which emits wavelengths between 620 and $710 \mathrm{~nm}$ if excited by light at about 400 nm. ${ }^{42}$ A natural biochemical precursor to hemoglobin, 5-ALA causes synthesis of PpIX in tissue. Protoporphyrin IX collects in cells because of the relatively low activity of ferrochelatase, the enzyme that converts PpIX to heme and then preferentially accumulates in tumor cells. ${ }^{5}$

Protoporphyrin IX fluorescence can delineate individual cells when visualized with confocal microscopy; however, the signal bleaches quickly while sampling with a point-scanning confocal microscope. Fluorescence induced by 5 -ALA decays to $36 \%$ within 25 minutes with 405-nm excitation (filtered light to match excitation wavelength); in contrast, with unfiltered wide-field illumination, it takes 87 minutes to reach the same level of decay. ${ }^{43}$ Confocal microscopes with greater acquisition rates, which deposit less excitation energy into samples, may prove more feasible for in vivo and immediate ex vivo imaging of PpIX. Protoporphyrin IX fluorescence collected with the VWCE could neither exactly delineate cells, nor provide histological information.

In our experiments, in vivo handheld confocal microscopy detected PpIX as bright pixels. Differences in the imaging appearance of normal brain and tumor tissue reflected the discrepant amount of bright pixels within the field of view, with significantly fewer pixels associated with normal tissue. Furthermore, the number of bright pixels varied from one tumor region to another, possibly reflecting differences in biological properties (for example, necrosis vs cellular tumor) and variable incorporation of the 5-ALA. The signal-to-noise limitation of the VWCE system also affected interpretation of images. Bright pixels detected in normal brain could be explained as PpIXpositive cells or as autofluorescence signal, although more bright pixels were detected within the tumors than outside them. The limited laser power of the handheld unit could also influence low-image quality. Furthermore, the optimal excitation of 5-ALA may be just beyond the range of the VWCE system. Published reports show the optimal excitation for PpIX is $405 \mathrm{~nm}$, while the handheld unit excitation wavelength is $488 \mathrm{~nm}$. Modification of the instrument might improve PpIX imaging parameters.

Imaging of PpIX fluorescence with the LSM revealed fluorescence within tumor tissue and minimal fluores- cence in normal brain. Imaging of samples using higher laser power was associated with the generation of autofluorescence signal, which was evident in both normal brain and tumor tissue. However, spectral imaging with the benchtop confocal microscope confirmed and differentiated PpIX fluorescence from autofluorescence.

\section{Rapid Fluorescent Dyes Suitable for Ex Vivo Imaging}

Acriflavine Hydrochloride and AO. Since the beginning of the 20th century, acridine family compounds have been well known for their pharmacological properties. ${ }^{19}$ Acriflavine hydrochloride has been used as a topical antiseptic, and acridine derivatives have been used for antimalarial and anticancer therapy. Pharmacological properties of these agents are closely related to reversible binding of these compounds to DNA regulatory enzymes. They cause frameshift mutations and inhibit DNA repair. Acridine compounds exhibit mutagenic and carcinogenic properties. ${ }^{19,24}$ Acriflavine hydrochloride has been used extensively for confocal endomicroscopy of the gastrointestinal tract. ${ }^{16,20,32,46}$ However, gastrointestinal mucosal cells show a rapid turnover rate, and AF-stained cells are rapidly replaced with new cells. ${ }^{7}$ In contrast, application of AF to intracranial tissue may cause long-lasting mutagenic effects due to slow or absent cell turnover. For this reason, the safety of $\mathrm{AO}$ and $\mathrm{AF}$ for in vivo human brain applications seems doubtful.

Acridine orange and $\mathrm{AF}$ in tumor regions function predominantly as intracellular fluorophores, highlighting the nucleus and its contents. ${ }^{6,38}$ They provide fluorescent staining within seconds of application. Tumor regions exhibit classic histological features (for example, atypia and pleomorphism). The absence of cytoplasmic staining allows visualization of important diagnostic features such as mitoses. Acriflavine hydrochloride also provides weak membrane staining, which helps identify cells visually. Both $\mathrm{AO}$ and AF stain extracellular matrix, thereby defining regional tissue architecture. Furthermore, imaging of normal brain using AO and the VWCE reveals typical histological features that closely correlate with those seen on conventional histological staining of the areas. Interestingly, some cells do not exhibit intracellular/intranuclear staining with AO (for example, Purkinje cells). Cortical neurons appeared darker than large round background cells. Neurons in the basal ganglia showed bright membrane staining, delineating a polygonal cell shape. In our study, AF and AO yielded the most informative and rapid images. These properties suggest these fluorophores have the potential for enabling rapid intraoperative ex vivo analysis of tissue.

The effect of "supplanting" FNa with AO could further help to determine subcellular structures of tumor cells imaged with FNa.

Cresyl Violet Acetate. Cresyl violet has been used extensively to stain nerve tissue because its basic chemical properties allow interaction with the acidic components of the neuronal cytoplasm, especially RNA-rich ribosomes. It is a nontoxic, clinically safe compound. ${ }^{14,17}$ In our study, it provided cytoplasmic fluorescence in tumor cells and cellular structures of normal brain (for example, ependyma of lateral ventricles). Because of the nonopti- 
mal coupling of excitation and emission spectra as well as the limited laser power of the VWCE system, images had relatively low signal-to-noise characteristics. Cellular features (for example, atypia, pleomorphism, and mitoses) were evident within the field of view. Although cellular areas could be identified, the device was unable to image extracellular components. With the benchtop microscope, CV more easily delineated tissue architecture and tumor boundaries.

Sulforhodamine 101. This dye has been reported to specifically label astrocytes in the rodent brain. It has been used extensively to label astrocytes both in vivo and ex vivo. ${ }^{4,27,28}$ The detailed mechanisms of its apparent selective fluorescence as well as its toxicity in in vivo use is still under investigation. Selective staining is achieved through local uptake and gap junction-mediated spread of SR101 following its application. ${ }^{4}$ We found that SR101 strongly labeled cells within frank tumor regions and identified cells that morphologically resembled astrocytes within normal brain and peritumoral regions.

\section{Normal Brain}

In our study, the VWCE system was effective at showing characteristic histoarchitectural patterns in different areas of normal brain, especially as demonstrated by the AO staining in swine brain tissue. Distinct histological features, such as neurons, glial cells, ependymal cells, and fiber tracts, could be identified on confocal images. Furthermore, the imaging of normal brain resulted in a frame of reference of normal cytoarchitecture used to compare with tumor tissue. Thus, handheld confocal microscopy can distinguish between normal brain and neoplastic tissue intraoperatively, which is important for instrument capability if fully implemented to help ensure completeness of glioma resection.

\section{Conclusions}

Our study findings suggest that handheld confocal microscopy with the Optiscan 5.1 VWCE system has the potential to be a valuable tool for in vivo clinical and research applications. Imaging with clinically approved fluorophores provides instant intraoperative histological information. Our previous limited clinical studies indicated the efficacy of in vivo imaging with $\mathrm{FNa}$ in providing cytoarchitectural information and, in some cases, histological diagnosis for a variety of intracranial lesions, mainly tumors. Using FNa systematically in the present study of a malignant murine glioma showed excellent image capture and resolution by the Optiscan 5.1 VWCE system and the ability to discriminate between tumor and nontumor regions. Although some cell types, such as multinucleated and mitotic cells, could be identified, exact identification of the various cell types in the field of view is challenging. With the VWCE system, the use of other fluorophores, such as $\mathrm{AO}, \mathrm{AF}$, and $\mathrm{CV}$, greatly enhances the imaging experience and yield of cellular information. Perhaps some fluorophores can be used in combination or sequentially, as shown by our $\mathrm{FNa}$ to $\mathrm{AO}$ observations, or can be used ex vivo within seconds of tissue sampling. Although these fluorophores can be used in vivo in animals, some cannot be used in patients with brain tumors. At $488 \mathrm{~nm}$, Optiscan 5.1 VWCE imaging of 5-ALA seems unreliable because of the suboptimal excitation range and rapid fluorescence bleaching. Improving laser power or combining other laser lines into the instrument may significantly improve the utility of the instrument. However, such modifications could affect the portability of confocal imaging, which is a significant advantage. The Optiscan 5.1 VWCE is a useful instrument for research applications. Developing additional compatible fluorophores for in vivo use in humans, such as specific tumor molecular labels, may increase use of the current instrument. Expert and blind assessment of a large training library of varied tissue images acquired methodically and systematically with many fluorophores will be crucial to the successful application of this promising microimaging technology.

Applying fluorescent techniques in the human brain to aid surgery or treat tumors is in its infancy, and what has been presented here are experimental data using preclinical techniques. Appropriate fluorophore use in the human brain remains relatively restricted and necessitates efforts to identify safe and effective fluorophores for in vivo use, although ex vivo use may initially be more practical. The potential advantages of cell-based imaging using a portable imaging tool in vivo to improve the location and limits of resection, with histological image interpretation on the fly, support continued development and exploration of this technology. Further preclinical and clinical trial investigations are needed to validate the use of these fluorophores and to define roles for this imaging technology for clinical human brain tumor applications.

\section{Disclosure}

This work was supported by a grant that supplied materials from Carl Zeiss Surgical, GmbH (P.N.). Carl Zeiss Surgical, GmbH had no input in the interpretation of imaging or histology. Barrow Neurological Institute has no financial or marketing relationship with Optiscan Pty., Ltd., or with Carl Zeiss Surgical, GmbH, for the technology described in this article. Other funding for this project came from the Newsome Endowment in Neurosurgery Research held by Dr. Preul and from a Barrow Neurological Foundation grant to Dr. Martirosyan. The authors report no conflict of interest concerning the materials or methods used in this study or the findings specified in this paper.

Author contributions to the study and manuscript preparation include the following. Conception and design: Preul, Martirosyan, Georges, Eschbacher, Cavalcanti, Nakaji. Acquisition of data: Martirosyan, Georges, Cavalcanti, Elhadi, Abdelwahab. Analysis and interpretation of data: Preul, Martirosyan, Georges, Eschbacher. Drafting the article: Martirosyan, Georges, Cavalcanti, Elhadi, Abdelwahab. Critically revising the article: Preul, Eschbacher, Scheck, Nakaji, Spetzler. Reviewed submitted version of manuscript: Preul, Scheck, Nakaji, Spetzler. Administrative/technical/ material support: Preul, Scheck, Nakaji, Spetzler. Study supervision: Preul, Scheck, Nakaji, Spetzler.

\section{References}

1. Ackerman LV, Ramirez GA: The indications for and limitations of frozen section diagnosis; a review of 1269 consecutive frozen section diagnoses. Br J Surg 46:336-350, 1959

2. Ammirati M, Vick N, Liao YL, Ciric I, Mikhael M: Effect of the extent of surgical resection on survival and quality of life 


\section{Handheld confocal microscopy with various rapid fluorescent dyes}

in patients with supratentorial glioblastomas and anaplastic astrocytomas. Neurosurgery 21:201-206, 1987

3. Ando T, Kobayashi E, Liao H, Maruyama T, Muragaki Y, Iseki $\mathrm{H}$, et al: Precise comparison of protoporphyrin IX fluorescence spectra with pathological results for brain tumor tissue identification. Brain Tumor Pathol 28:43-51, 2011

4. Appaix F, Girod S, Boisseau S, Römer J, Vial JC, Albrieux M, et al: Specific in vivo staining of astrocytes in the whole brain after intravenous injection of sulforhodamine dyes. PLoS ONE 7:e35169, 2012

5. Black PM: Brain tumors. Part 1. N Engl J Med 324:1471-1476, 1991

6. Boyd V, Cholewa OM, Papas KK: Limitations in the use of fluorescein diacetate/propidium iodide (FDA/PI) and cell permeable nucleic acid stains for viability measurements of isolated islets of Langerhans. Curr Trends Biotechnol Pharm 2:66-84, 2008

7. Creamer B: The turnover of the epithelium of the small intestine. Br Med Bull 23:226-230, 1967

8. Debarbieux S, Depaepe L, Poulalhon N, Balme B, Dalle S, Thomas L: Reflectance confocal microscopy accurately discriminates between benign and malignant melanocytic lesions exhibiting a 'dermoscopic island.' J Eur Acad Dermatol Venereol 27:e159-e165, 2013

9. Devaux BC, O'Fallon JR, Kelly PJ: Resection, biopsy, and survival in malignant glial neoplasms. A retrospective study of clinical parameters, therapy, and outcome. J Neurosurg 78: 767-775, 1993

10. Diaspro A, Chirico G, Collini M: Two-photon fluorescence excitation and related techniques in biological microscopy. $\mathbf{Q}$ Rev Biophys 38:97-166, 2005

11. Drobizhev M, Makarov NS, Tillo SE, Hughes TE, Rebane A: Two-photon absorption properties of fluorescent proteins. Nat Methods 8:393-399, 2011

12. Eschbacher J, Martirosyan NL, Nakaji P, Sanai N, Preul MC, Smith KA, et al: In vivo intraoperative confocal microscopy for real-time histopathological imaging of brain tumors. Clinical article. J Neurosurg 116:854-860, 2012

13. Fuchs FS, Zirlik S, Hildner K, Frieser M, Ganslmayer M, Schwarz S, et al: Fluorescein-aided confocal laser endomicroscopy of the lung. Respiration 81:32-38, 2011

14. George M, Meining A: Cresyl violet as a fluorophore in confocal laser scanning microscopy for future in-vivo histopathology. Endoscopy 35:585-589, 2003

15. Gerganov VM, Samii A, Akbarian A, Stieglitz L, Samii M, Fahlbusch R: Reliability of intraoperative high-resolution 2D ultrasound as an alternative to high-field strength MR imaging for tumor resection control: a prospective comparative study. Clinical article. J Neurosurg 111:512-519, 2009

16. Goetz M, Memadathil B, Biesterfeld S, Schneider C, Gregor $\mathrm{S}$, Galle PR, et al: In vivo subsurface morphological and functional cellular and subcellular imaging of the gastrointestinal tract with confocal mini-microscopy. World J Gastroenterol 13:2160-2165, 2007

17. Goetz M, Toermer T, Vieth M, Dunbar K, Hoffman A, Galle PR, et al: Simultaneous confocal laser endomicroscopy and chromoendoscopy with topical cresyl violet. Gastrointest Endose 70:959-968, 2009

18. Gong H, Kovar JL, Baker B, Zhang A, Cheung L, Draney DR, et al: Near-infrared fluorescence imaging of mammalian cells and xenograft tumors with SNAP-tag. PLoS ONE 7:e34003, 2012

19. Hashiguchi S, Kusuzaki K, Murata H, Takeshita H, Hashiba $\mathrm{M}$, Nishimura T, et al: Acridine orange excited by low-dose radiation has a strong cytocidal effect on mouse osteosarcoma. Oncology 62:85-93, 2002

20. Haxel BR, Goetz M, Kiesslich R, Gosepath J: Confocal endomicroscopy: a novel application for imaging of oral and oropharyngeal mucosa in human. Eur Arch Otorhinolaryngol 267:443-448, 2010
21. Kantelhardt SR, Leppert J, Krajewski J, Petkus N, Reusche E, Tronnier VM, et al: Imaging of brain and brain tumor specimens by time-resolved multiphoton excitation microscopy ex vivo. Neuro Oncol 9:103-112, 2007

22. Kuroiwa T, Kajimoto Y, Ohta T: Development and clinical application of near-infrared surgical microscope: preliminary report. Minim Invasive Neurosurg 44:240-242, 2001

23. Lacroix M, Abi-Said D, Fourney DR, Gokaslan ZL, Shi W, DeMonte F, et al: A multivariate analysis of 416 patients with glioblastoma multiforme: prognosis, extent of resection, and survival. J Neurosurg 95:190-198, 2001

24. Martin JP, Logsdon N: Oxygen radicals mediate cell inactivation by acridine dyes, fluorescein, and lucifer yellow CH. Photochem Photobiol 46:45-53, 1987

25. Martirosyan NL, Cavalcanti DD, Eschbacher JM, Delaney PM, Scheck AC, Abdelwahab MG, et al: Use of in vivo near-infrared laser confocal endomicroscopy with indocyanine green to detect the boundary of infiltrative tumor. Laboratory investigation. J Neurosurg 115:1131-1138, 2011

26. Neumann H, Kiesslich R, Wallace MB, Neurath MF: Confocal laser endomicroscopy: technical advances and clinical applications. Gastroenterology 139:388-392.e2, 2010

27. Nimmerjahn A, Helmchen F: In vivo labeling of cortical astrocytes with sulforhodamine 101 (SR101). Cold Spring Harb Protoc 2012:326-334, 2012

28. Nimmerjahn A, Kirchhoff F, Kerr JN, Helmchen F: Sulforhodamine 101 as a specific marker of astroglia in the neocortex in vivo. Nat Methods 1:31-37, 2004

29. Nitta T, Sato K: Prognostic implications of the extent of surgical resection in patients with intracranial malignant gliomas. Cancer 75:2727-2731, 1995

30. Novis DA, Zarbo RJ: Interinstitutional comparison of frozen section turnaround time. A College of American Pathologists Q-Probes study of 32868 frozen sections in 700 hospitals. Arch Pathol Lab Med 121:559-567, 1997

31. Olson ES, Jiang T, Aguilera TA, Nguyen QT, Ellies LG, Scadeng M, et al: Activatable cell penetrating peptides linked to nanoparticles as dual probes for in vivo fluorescence and MR imaging of proteases. Proc Natl Acad Sci U S A 107: 4311-4316, 2010

32. Polglase AL, McLaren WJ, Skinner SA, Kiesslich R, Neurath MF, Delaney PM: A fluorescence confocal endomicroscope for in vivo microscopy of the upper- and the lower-GI tract. Gastrointest Endosc 62:686-695, 2005

33. Rostomily RC, Spence AM, Duong D, McCormick K, Bland M, Berger MS: Multimodality management of recurrent adult malignant gliomas: results of a phase II multiagent chemotherapy study and analysis of cytoreductive surgery. Neurosurgery 35:378-388, 1994

34. Salem N, Balkman JD, Wang J, Wilson DL, Lee Z, King CL, et al: In vivo imaging of schistosomes to assess disease burden using positron emission tomography (PET). PLoS Negl Trop Dis 4:e827, 2010

35. Sanai N, Eschbacher J, Hattendorf G, Coons SW, Preul MC, Smith KA, et al: Intraoperative confocal microscopy for brain tumors: a feasibility analysis in humans. Neurosurgery 68 (2 Suppl Operative):ons282-ons290, 2011

36. Sanai N, Snyder LA, Honea NJ, Coons SW, Eschbacher JM, Smith KA, et al: Intraoperative confocal microscopy in the visualization of 5-aminolevulinic acid fluorescence in low-grade gliomas. Clinical article. J Neurosurg 115:740-748, 2011

37. Sankar T, Delaney PM, Ryan RW, Eschbacher J, Abdelwahab M, Nakaji P, et al: Miniaturized handheld confocal microscopy for neurosurgery: results in an experimental glioblastoma model. Neurosurgery 66:410-418, 2010

38. Sharma VK, Sahare PD, Rastogi RC, Ghoshal SK, Mohan D: Excited state characteristics of acridine dyes: acriflavine and acridine orange. Spectrochim Acta A Mol Biomol Spectrosc 59:1799-1804, 2003 


\section{N. L. Martirosyan et al.}

39. Sonn GA, Jones SN, Tarin TV, Du CB, Mach KE, Jensen KC, et al: Optical biopsy of human bladder neoplasia with in vivo confocal laser endomicroscopy. J Urol 182:1299-1305, 2009

40. Steinmeier R, Fahlbusch R, Ganslandt O, Nimsky C, Buchfelder M, Kaus M, et al: Intraoperative magnetic resonance imaging with the magnetom open scanner: concepts, neurosurgical indications, and procedures: a preliminary report. Neurosurgery 43:739-748, 1998

41. Stummer W, Novotny A, Stepp H, Goetz C, Bise K, Reulen HJ: Fluorescence-guided resection of glioblastoma multiforme by using 5-aminolevulinic acid-induced porphyrins: a prospective study in 52 consecutive patients. J Neurosurg 93:10031013, 2000

42. Stummer W, Pichlmeier U, Meinel T, Wiestler OD, Zanella F, Reulen HJ: Fluorescence-guided surgery with 5-aminolevulinic acid for resection of malignant glioma: a randomised controlled multicentre phase III trial. Lancet Oncol 7:392-401, 2006

43. Stummer W, Stocker S, Wagner S, Stepp H, Fritsch C, Goetz C, et al: Intraoperative detection of malignant gliomas by 5 -aminolevulinic acid-induced porphyrin fluorescence. Neurosurgery 42:518-526, 1998

44. Tan J, Quinn MA, Pyman JM, Delaney PM, McLaren WJ: Detection of cervical intraepithelial neoplasia in vivo using confocal endomicroscopy. BJOG 116:1663-1670, 2009

45. van den Broek FJ, van Es JA, van Eeden S, Stokkers PC, Ponsioen CY, Reitsma JB, et al: Pilot study of probe-based confocal laser endomicroscopy during colonoscopic surveillance of patients with longstanding ulcerative colitis. Endoscopy 43: 116-122, 2011

46. Venkatesh K, Cohen M, Evans C, Delaney P, Thomas S, Taylor $\mathrm{C}$, et al: Feasibility of confocal endomicroscopy in the diagnosis of pediatric gastrointestinal disorders. World J Gastroenterol 15:2214-2219, 2009

47. Wallace MB, Meining A, Canto MI, Fockens P, Miehlke S, Roesch T, et al: The safety of intravenous fluorescein for confocal laser endomicroscopy in the gastrointestinal tract. Aliment Pharmacol Ther 31:548-552, 2010

48. Wood JR, Green SB, Shapiro WR: The prognostic importance of tumor size in malignant gliomas: a computed tomographic scan study by the Brain Tumor Cooperative Group. J Clin Oncol 6:338-343, 1988

49. Zipfel WR, Williams RM, Webb WW: Nonlinear magic: multiphoton microscopy in the biosciences. Nat Biotechnol 21: 1369-1377, 2003

Manuscript submitted October 15, 2013.

Accepted November 27, 2013.

Please include this information when citing this paper: DOI: 10.3171/2013.11.FOCUS13486.

Address correspondence to: Mark C. Preul, M.D., c/o Neuroscience Publications, Barrow Neurological Institute, St. Joseph's Hospital and Medical Center, 350 W. Thomas Rd., Phoenix, AZ 85013. email: Neuropub@dignityhealth.org. 